PROCEEDINGS OF THE

AMERICAN MATHEMATICAL SOCIETY

Volume 129, Number 10, Pages 2919-2921

S 0002-9939(01)05896-8

Article electronically published on February 22, 2001

\title{
THE NATURAL MAXIMAL OPERATOR ON BMO
}

\author{
WINSTON OU
}

(Communicated by Christopher D. Sogge)

\begin{abstract}
We introduce a generalization of the Hardy-Littlewood maximal operator, the natural maximal operator $M^{\natural}$, in some sense the maximal operator which most naturally commutes pointwise with the logarithm on $A^{\infty}$. This commutation reveals the behavior of $M: A^{\infty} \rightarrow A^{1}$ to directly correspond to that of $M^{\natural}: B M O \rightarrow B L O$; the boundedness of $M: B M O \rightarrow B L O$ is an immediate consequence.
\end{abstract}

In 1981 Bennett, DeVore, and Sharpley 1 proved the Hardy-Littlewood maximal operator $M$ to be bounded on $B M O$. Later [4], this was improved to yield $M: B M O \rightarrow B L \mathrm{O}^{1}$ and furthermore was shown to be intimately related to the observation that $M$ maps $A^{\infty}$ into $A^{1}$. In this paper we shall clarify this relationship and in fact demonstrate a precise equivalence.

Let us begin by defining the natural maximal operator $M^{\natural}$ by

$$
M^{\natural} f(x)=\sup _{Q \ni x} \frac{1}{|Q|} \int_{Q} f .
$$

Of course, $M(f)(x)=M^{\natural}(|f|)(x)$; so to prove the boundedness of $M$ it suffices to show that of $M^{\natural}$. This is easily accomplished with the following commutation lemma, which allows us to pass between the language of $A^{\infty}$ and that of $B M O$.

Lemma. For $w \in A^{\infty}, 0 \leq\left[\log M^{\natural}-M^{\natural} \log \right] w(x) \leq \log A^{\infty}(w) ! 2$

Proof. By Jensen's inequality and the reverse inequality for $A^{\infty}$,

$$
\frac{1}{|Q|} \int_{Q} w \leq A^{\infty}(w) e^{\frac{1}{|Q|} \int_{Q} \log w} \leq A^{\infty}(w) \frac{1}{|Q|} \int_{Q} w
$$

Take the supremum over all $Q \ni x$ and then take log.

Theorem. $M^{\natural}$ maps BMO boundedly into BLO.

Received by the editors February 3, 2000.

2000 Mathematics Subject Classification. Primary $42 \mathrm{~B} 25$.

The author was partially supported by an NSF Graduate Fellowship. Many thanks to Professor R. Fefferman for his unflagging encouragement and repeated proofreading, and also to Professor C. Kenig for checking over the argument. Any errors are of course the sole property of the author.

${ }^{1}$ Recall that $B L O$ denotes the functions of bounded lower oscillation, i.e. $f$ such that over all cubes $Q, \frac{1}{|Q|} \int_{Q} f-\inf _{Q} f \leq C$; we denote by $\|f\|_{B L O}$ the smallest such $C$.

${ }^{2} A^{\infty}(w)$ denotes the smallest $C$ such that for all cubes $Q, \frac{1}{|Q|} \int_{Q} w \leq C e^{\frac{1}{|Q|} \int_{Q} \log w}$. 
Proof. Let $\phi \in B M O$. As a consequence of the John-Nirenberg Inequality, $\phi(x)=$ $\frac{\|\phi\|_{*}}{c_{n}} \log w(x)$ for some $w \in A^{2}$, with $A^{2}(w) \leq \sqrt{e}$ and $c_{n}=\frac{1}{2^{n+1} e}$, where $n$ denotes the dimension. Thus, by the lemma,

$$
M^{\natural} \phi(x)=\frac{\|\phi\|_{*}}{c_{n}}[\log M w(x)+b(x)]
$$

for some $\|b\|_{\infty} \leq 1$. Let's consider the $\log M w$ term: $M$ maps $A^{\infty}$ into $A^{1}$, and $\log$ maps $A^{1}$ into $B L O$; so it is in $B L O$. Further, its norm depends on $A^{1}(M w)$, which depends on the reverse Hölder class and norm of $w$, which in turn depend on the $A^{p}$ class and $A^{p}$ norm of $w$. As noted above, these depend on nothing; thus $\left\|M^{\natural} \phi\right\|_{B L O} \leq C_{n}\|\phi\|_{*}$.

Corollary. $M$ maps BMO boundedly into $B L O 3$

Proof. $M f=M^{\natural}(|f|) \in B L O$, and $\|M f\|_{B L O}=\left\|M^{\natural}(|f|)\right\|\left\|_{B L O} \leq 2 C_{n}\right\| f \|_{*}$.

Notice that above, the set inclusion $M\left(A^{\infty}\right) \subset A^{1}$ implies $M^{\natural}(B M O) \subset B L O$, and the dependence of $A^{1}(M w)$ on $A^{p}(w)$ and $p$ implies the boundedness of $M^{\natural}$. Repeated application of the commutation lemma, combined with the observation that $\phi \in B L O$ if and only if $M^{\natural} \phi(x) \leq \phi(x)+\|\phi\|_{B L O}$, yields the following converse (assuming the weak result that $M\left(A^{\infty}\right) \subset A^{\infty}$ with $A^{\infty}(M w)$ dependent on $A^{p}(w)$ and $p$ ):

Theorem. $M^{\natural}: B M O \rightarrow B L O$ bounded implies $M\left(A^{\infty}\right) \subset A^{1}$ with $A^{1}(M w)$ dependent on $A^{p}(w)$ and $p$.

Proof. Let $w \in A^{\infty}$. The commutation lemma applied to $w$ implies

$$
e^{M^{\natural} \log w(x)} \leq M w(x) \leq A^{\infty}(w) e^{M^{\natural} \log w(x)} ;
$$

applied twice to $M w$ it yields

$$
e^{M^{\natural} M^{\natural} \log w(x)} \leq M M w(x) \leq A^{\infty}(w) A^{\infty}(M w) e^{M^{\natural} M^{\natural} \log w(x)} .
$$

By hypothesis, $M^{\natural} \log w \in B L O$; thus

$$
M M w(x) \approx e^{M^{\natural} M^{\natural} \log w(x)} \leq e^{M^{\natural} \log w(x)+\left\|M^{\natural} \log w\right\|_{B L O}} \approx e^{\left\|M^{\natural} \log w\right\|_{B L O}} M w(x),
$$

i.e., $M w \in A^{1}$, with $A^{1}(M w) \leq A^{\infty}(w) A^{\infty}(M w) e^{C_{n}\|\log w\|_{*}}$.

In other words, the behavior of $M$ on $A^{\infty}$ corresponds exactly to that of $M^{\natural}$ on $B M O$. For amusement, one can adapt the original proof of Bennett, DeVore, and Sharpley to prove directly the bound for $M^{\natural}$ and thus obtain a new, if unwieldy, proof of $M: A^{\infty} \rightarrow A^{1}$.

\section{REFERENCES}

[1] C. Bennett, R. DeVore, and R. Sharpley, Weak- $L^{\infty}$ and $B M O$, Annals of Mathematics, 113 (1981), 601-611. MR 82h:46047

[2] F. Chiarenza and M. Frasca, Morrey spaces and Hardy-Littlewood maximal function, Rendiconti Di Matematica e Delle Sue Applicazioni, ser. 7, 7 (1987), 273-279. MR 90f:42017

[3] R. Coifman and R. Rochberg, Another characterization of BMO, Proceedings of the American Mathematical Society, 79 (1980), 249-254. MR 81b:42067

\footnotetext{
${ }^{3}$ Added in proof: Chiarenza and Frasca 2] have also proved this bound directly using the John-Nirenberg inequality and the fact that $(M f)^{1 / 2} \in A^{1}$ for $f \in L_{l o c}^{1}\left(\mathbb{R}^{n}\right)[3$, though without the commutation or noticing the connection with the behavior on $A^{\infty}$.
} 
[4] D. Cruz-Uribe and C.J. Neugebauer, The structure of the reverse Hölder classes, vol. 347, Transactions of the American Mathematical Society, 8 (1995), 2941-2960. MR 95m:42026

[5] J. Garcia-Cuerva and J. L. Rubio de Francia, Weighted Norm Inequalities and Related Topics, North-Holland Mathematics Studies, vol. 116, North-Holland, Amsterdam, 1985. MR 87d:42023

[6] E. Stein, Harmonic Analysis: Real-Variable Methods, Orthogonality, and Oscillatory Integrals, Princeton Mathematical Series, vol. 43, Princeton University Press, Princeton, 1993. MR 95c:42002

Department of Mathematics, University of Chicago, Chicago, Illinois 60637

E-mail address: wcwou@math.uchicago.edu 